\title{
Erratum
}

\section{The effect of HII regions on rotation measure of pulsars}

\author{
D. Mitra ${ }^{1}$, R. Wielebinski ${ }^{1}$, M. Kramer ${ }^{2}$, and A. Jessner ${ }^{1}$ \\ 1 Max-Planck Institute für Radioastronomie, Auf dem Hügel 69, 53121 Bonn, Germany \\ 2 University of Manchester, Jodrell Bank Observatory, Macclesfield SK11 9DL, UK \\ A\&A, 398, 993-1005 (2003), DOI: 10.1051/0004-6361:20021702
}

Key words. errata, addenda, Galaxy: structure - magnetic fields - ISM: HII regions - stars: pulsars: general

At the beginning of Sect. 5, the following statement is missing:

The paucity of pulsars beyond a distance of $\sim 6 \mathrm{kpc}$ forces us to rely on the $R M \mathrm{~s}$ of extragalactic sources to study the magnetic field beyond the Perseus arm. As discussed in Brown (2002) and clarified further in Brown et al. (2003, in preparation), one needs extragalactic sources that are confined to a narrow Galactic latitude range.

\section{References}

Brown, J. C. 2002, Ph.D. Thesis, University of Calgary 